\title{
Influence of a combined bearing on the buildup rate of a wellbore trajectory control tool
}

\author{
Lei SHI*, Jiangang WANG*, Zuowei YANG*, Kai LUO*, Hong ZHANG* and Ding FENG* \\ * School of Mechanical Engineering, Yangtze University \\ 1 Nanhuan Road, Hubei, Jingzhou 434023, China \\ E-mail: fengd0861@163.com
}

Received: 31 July 2020; Revised: 5 October 2020; Accepted: 20 November 2020

\begin{abstract}
Conventional methods cannot accurately predict buildup rate. In this study, an algorithm was developed to predict the geometric deflection rate of the tool considering the influence of the radial displacement of a combined bearing. Three-point geometry was used to calculate the buildup rate and its relationship with the radial displacement of the combined bearing. The relation between the maximum spindle deflection and the load of the combined bearing was also determined by treating the spindle as a statically indeterminate beam. To consider the internal structural parameters of the combined bearing, a mathematical relationship was obtained between its load and radial displacement with the Stribeck maximum rolling element load formula and Palmgren roller bearing displacement formula. The influence of the structural parameters of the combined bearing on the buildup rate was analyzed, and the results showed that the buildup rate can be increased by increasing the thickness and pre-compression of the disc spring and decreasing its diameter ratio and the radial clearance of the self-aligning roller bearing. An experimental device was used to obtain test results with different maximum spindle deflection values. In particular, the error was less than $1 \%$ when the maximum spindle deflection was more than $4 \mathrm{~mm}$.
\end{abstract}

Keywords : Downhole directional drilling equipment, Point-the-bit rotary steerable tool, Combined bearing, Buildup rate, Mathematical model

\section{Introduction}

With the continuous development of point-the-bit rotary steerable tools, the tool's deflection rate needs to be predicted with ever greater accuracy. For example, precision directional drilling, which includes the excavation of directional wells and horizontal wells, needs to accurately hit small target areas. There is an urgent demand to improve the precision of the predicted deflection rate. At present, nearly ten petroleum technology service companies (e.g., Baker Hughes, Schlumberger, and Halliburton) have developed high-performance rotary steering drilling systems ( Li et al.,2020; Dong et al.,2015;Zhang,2016;Minett-Smith et al.,2012;Li,2014). However, it is difficult for ordinary downhole directional drilling equipment to predict the deflection rate with high accuracy in the case of spindles. Consequently, researchers around the world have focused on developing a rotary guidance tool that can accurately control the buildup rate and accurately predict the buildup rate considering spindle deflection(Hong et al.,2014;Wang et al.,2012;Feng et al.,2017; Shi et al.,2020).

Many researchers have used the geometric relationship between the short length and relatively high rigidity of the steering tool assembly to predict the tool's deflection rate and have achieved remarkable results. Karlsson and his group ( Karlsson and Brassfield,1985;Karlsson et al.,1989) used the fact that wellbore trajectories are similar to arcs to propose a three-point geometry for predicting the buildup rate, and their approach has become widely used. Based on this, Hassen and MacDonald (Hassen and MacDonald,1990) analyzed the buildup rate of double-bend drilling tools and proposed the double-radius method for predicting the buildup rate. Wang et al. (Wang et al.,1994) and Shuai et al. (Shi et al.,2014) studied the above method for predicting the deflection rate and expanded the application range. Su et al. (Su and 
Tang,2002) studied the equivalent relationship between the deflection rates of multi-bend and single-bend drilling tools. To consider the influence of the bending position on the deflection rate of the steering drilling tool, Liu and his group (Liu,2005; Liu et al.,2004) proposed a modified three-point fixed circle method. Sun et al. ( Kong and Li,2016) considered the buildup rate of downhole directional drilling equipment with undergauge stabilizers. The effective drilling force (Gong and Huang,2016) can also be used to predict the buildup rate.

However, the above prediction formulas for calculating the buildup rate all assume that the bearing does not shift and do not consider the load distribution of the bearing. Consequently, they are not suitable for accurately calculating the buildup rate of a spindle with a large deflection and heavy load. No theoretical formula has yet been reported for calculating the buildup rate of a wellbore trajectory control tool under a heavy load. This has consequently led to a lack of a theoretical basis for designing the structural parameters of downhole directional drilling equipment while considering the bearing behavior to meet accuracy requirements. Instead, such equipment is developed and modified according to experience and bench tests, which has resulted in poor tool-oriented precision control capabilities and greatly increased R\&D and manufacturing costs. Therefore, the influence of the combined bearing on the buildup rate of a rotary steerable tool needs to be considered.

The wellbore trajectory control tool is a type of point-the-bit rotary steerable tool. It is mainly used to control the bit pointing, adjust and change the wellbore trajectory formed by bit drilling. Its main working principle is to generate controllable elastic deformation of tool spindle through eccentric mechanism. The deformation of the spindle causes the end connected bit to deflect. In this study, a combined bearing was used to support the deformation and rotation of the spindle in wellbore trajectory control tool. The internal load distribution of the combined bearing during spindle deformation was analyzed, and a prediction model of the buildup rate was established to consider the influence of the working conditions and structural parameters on the buildup rate. The model was then tested and verified.

\section{Mathematical model of the deflection rate for the wellbore trajectory control tool 2.1 Working principle of the wellbore trajectory control tool}

The wellbore trajectory control tool's buildup rate is controlled by the deformation of its spindle. The working principle is shown in Fig.1. The buildup rate of the wellbore trajectory control tool refers to the full bending angle of the borehole formed in the buildup drilling footage. The influencing factors of the buildup rate are very complex and are related to not only the structural parameters of the tool, eccentricity, and combined bearing but also the weight on bit, accuracy of the assembly, and structure of the bottomhole assembly (BHA). The main working principle is to control the maximum deflection $\omega$ of the spindle. With the support of the combined bearing, the axis of the drill bit is deflected at the angle $\theta$ to control the tool's buildup rate. The combined bearing structure is shown in Fig.2. The outer ring raceways of the spherical thrust roller bearing and self-aligning roller bearing both constrain the joint bearing, which supports the rotation of the spindle around its axis and the deflection of the spindle. When the tool spindle is bent and a bit weight is applied, the combined bearing bears a large load. In particular, a radial load changes the axis of the rotation, which changes the actual deflection angle of the drill axis and affects the tool's buildup rate. A method was established for calculating the geometric deflection rate of the wellbore trajectory control tool considering the influence of the combined bearing.

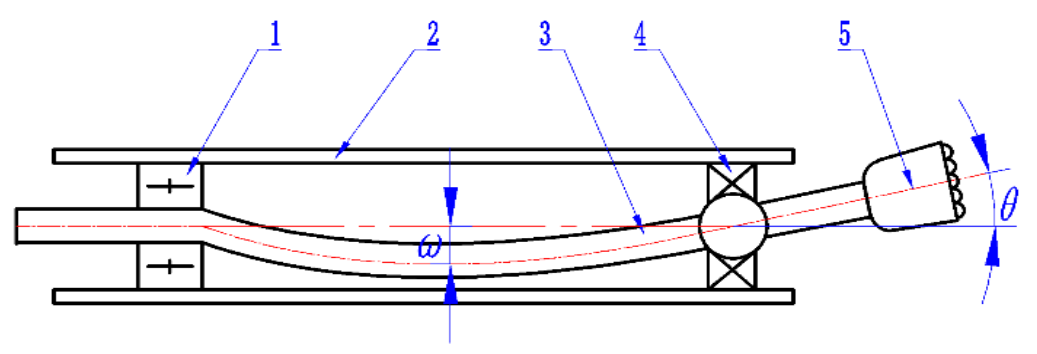

Fig. 1 Working principle of the buildup rate with the wellbore trajectory control tool: (1) upper bearing, (2) tool case, (3) spindle, (4) combined bearing, and (5) bit. 


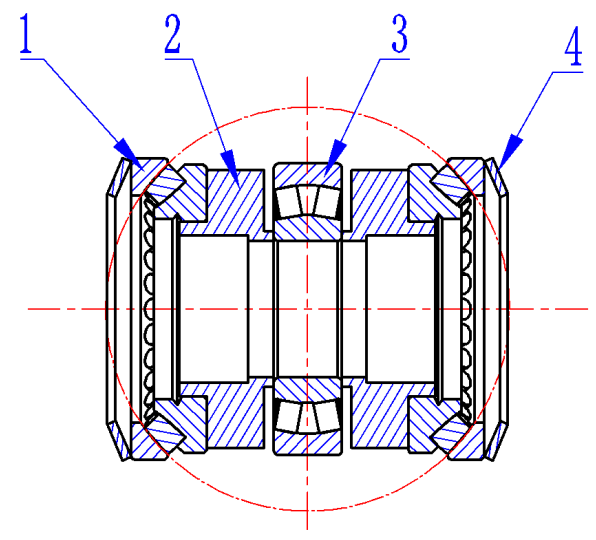

Fig. 2 Structure of the combined bearing for the wellbore trajectory control tool: (1) spherical thrust roller bearing, (2) bearing washer, (3) self-aligning roller bearing, and (4) disk spring.

\subsection{Mathematical model of the geometric deflection rate with the wellbore trajectory control tool}

During drilling, stabilizers are usually installed at the upper and lower ends of the wellbore trajectory control tool to stabilize the drilling direction. In this study three-point geometry was used to calculate the buildup rate. The geometric center of the upper stabilizer, geometric center of the lower stabilizer, and center of the bit diameter surface were taken as fixed points to establish the coordinate system, as shown in Fig.3. The wellbore trajectory control tool causes the tool spindle to bend and form the deflection angle as well as the bending elbow point. The coordinate parameters are as follows:

$$
\left\{\begin{array}{l}
y_{2}=L_{1} \\
x_{3}=L_{3} \sin \gamma \\
y_{3}=L_{1}+L_{2}+L_{3} \cos \gamma
\end{array}\right.
$$

where L1 is the distance from the drill bit to the lower stabilizer (m), L2 is the distance from the bending elbow point to the lower stabilizer $(\mathrm{m}), \mathrm{L} 3$ is the distance from the upper stabilizer point to the bending elbow (m), and $\gamma$ is the deflection angle of the drill $\left(^{\circ}\right)$. The buildup rate can be obtained by using the three-point circular method and cosine theorem, as shown in Eq. (2).

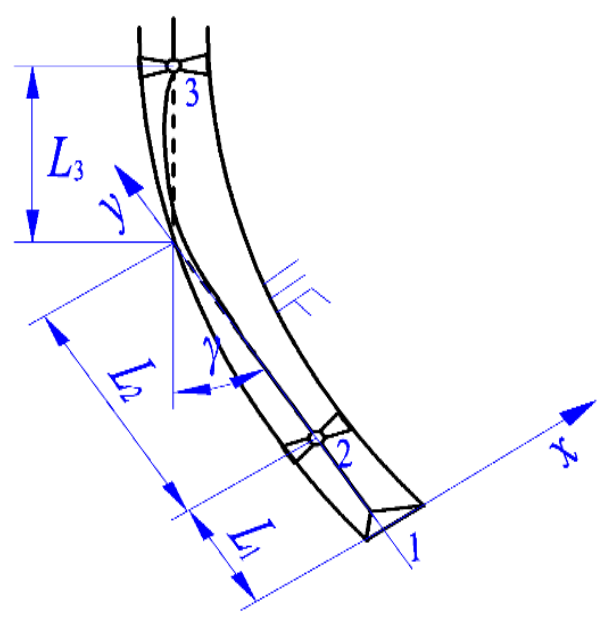

Fig.3 Schematic diagram of the buildup rate with the wellbore trajectory control tool: (1) bit, (2) lower stabilizer, (3) upper stabilizer. 


$$
k_{\mathrm{a}}=\frac{60 L_{3} \sin \gamma}{\sqrt{L_{1}^{2} L_{3}^{2} \sin ^{2} \gamma+\left[L_{2}^{2}+2 L_{2} L_{3} \cos \gamma+L_{3}^{2}+L_{1}\left(L_{2}+L_{3} \cos \gamma\right)\right]^{2}}}
$$

where $\mathrm{k}_{\mathrm{a}}$ is the buildup rate $\left({ }^{\circ} / 30 \mathrm{~m}\right)$. The diameter of the spindle is much less than its length, so it is a slender rod. When it is necessary for the tool to deviate, the built-in offset device offsets the spindle along the specified direction. At this time, the main axis of the tool can be regarded as a deflection curve. Because the upper bearing mainly limits the radial displacement of the tool spindle and bears the radial load and torque, its displacement is small, and the influence on the angle of the combined bearing can be ignored. The working condition of the tool is complex underground. To simplify the calculation, the following assumptions were made: (1) the effects of the drilling fluid and bottomhole temperature do not need to be considered; (2) the interaction between the bit and formation can be ignored, and the weight on the bit and torque are not considered to have an influence; and (3) the influence of the rigidity of the tool shell does not need to be considered. Based on the above assumptions, the upper bearing was set as the origin of a rectangular coordinate system on a plane with the $\mathrm{x}$-axis along the combined bearing direction and the $\mathrm{y}$-axis along the vertical direction in Fig.4. The static model of the spindle affected by the radial displacement of the combined bearing as shown below:

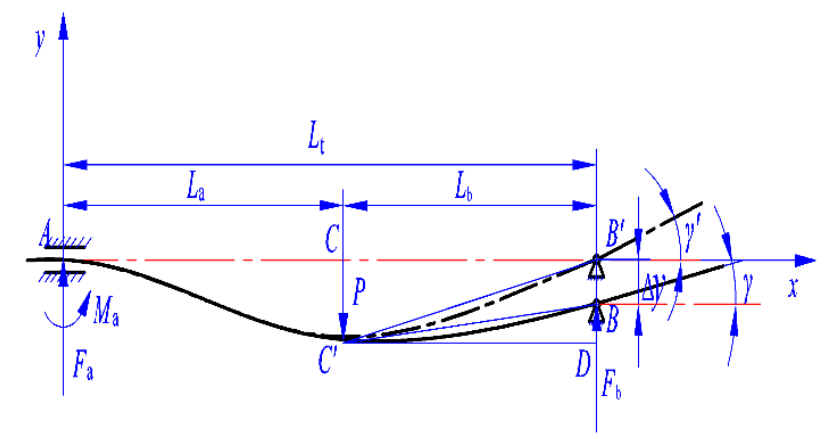

Fig. 4 Static model of the radial displacement of the combined bearing affecting the spindle.

Because the number of unknown constraints exceeds the number of independent static equilibrium equations, the mechanical model of the main shaft is a statically indeterminate beam problem. According to the deformation coordination condition of the beam, the supplementary equation is as follows:

$$
\omega_{\mathrm{B}}=\left(\omega_{\mathrm{B}}\right)_{P}+\left(\omega_{\mathrm{B}}\right)_{F_{\mathrm{b}}}=0
$$

where $\omega_{\mathrm{B}}$ is the deflection at point $\mathrm{B}(\mathrm{m}),\left(\omega_{\mathrm{B}}\right)_{P}$ is the deflection at point $\mathrm{B}$ under force $\mathrm{P}$ alone $(\mathrm{m})$, and $\left(\omega_{\mathrm{B}}\right)_{F_{\mathrm{b}}}$ is the deflection at point $\mathrm{B}$ under force $F_{\mathrm{b}}$ alone $(\mathrm{m})$.

If the radial displacement of the combined bearing is not considered, the superposition principle can be used to derive the relationships between the load at the combined bearing $(\mathrm{Fb})$ and the deflection angle $\left(\gamma^{\prime}\right)$ of the drill bit and between the offset load $(\mathrm{P})$ and maximum deflection $(\omega)$ :

$$
\begin{aligned}
& F_{\mathrm{b}}=\frac{P L_{\mathrm{a}}^{2}\left(2 L_{\mathrm{t}}+L_{\mathrm{b}}\right)}{2 L_{\mathrm{t}}^{3}} \\
& \gamma^{\prime}=-\frac{180 P L_{\mathrm{b}} L_{\mathrm{a}}^{2}}{4 E I L_{\mathrm{t}} \pi} \\
& P=\frac{12 \omega E I L_{\mathrm{t}}^{3}}{L_{\mathrm{a}}^{2} L_{\mathrm{b}}^{2}\left(3 L_{\mathrm{b}}^{2}+2 L_{\mathrm{a}} L_{\mathrm{b}}-3 L_{\mathrm{t}}^{2}\right)}
\end{aligned}
$$

where $L_{\mathrm{a}}$ is the distance from the elbow point to the upper bearing $(\mathrm{m}), L_{\mathrm{b}}$ is the distance from the elbow point 
to the combined bearing $(\mathrm{m}), L_{\mathrm{t}}$ is the distance from the upper bearing to the combined bearing (m), $P$ is the offset load applied to the spindle by the offset mechanism $(\mathrm{N}), E$ is the elastic modulus of the spindle (Pa), $\omega$ is the maximum deflection of the spindle controlled by the offset mechanism (mm), $I$ is the moment of inertia given by

$$
I=\frac{\pi}{64} D_{\mathrm{a}}^{4}(\mathrm{~m} 4) \text {, and } D_{\mathrm{a}} \text { is the diameter of the tool spindle (mm). }
$$

The combined bearing of the tool bears a large load when the buildup rate is increasing. Because of the radial clearance, the rotary axis also experiences a radial displacement $(\Delta y)$ that affects the deflection angle of the bit. The bit's deflection angle is generally very small and is given by $\tan \gamma=\gamma, \tan \gamma^{\prime}=\gamma^{\prime}$. Therefore, the bit deflection angle

$(\gamma)$ considering the influence of the radial displacement $(\Delta y)$ of the combined bearing is as follows:

$$
\gamma=\frac{\omega-\Delta y}{\omega} \gamma^{\prime}=\frac{180 P L_{\mathrm{b}} L_{\mathrm{a}}^{2}(\omega-\Delta y)}{4 \pi \omega E I L_{\mathrm{t}}}
$$

$\gamma$ can be substituted into Eq. (2). Because the bit deflection angle is very small, $\sin \gamma=\gamma, \sin ^{2} \gamma=0$, and $\cos \gamma=1$. Introducing Eq. (7) into eq. (2), eq. (8) is obtained. Considering the influence of the radial displacement of the combined bearing, the geometric deflection rate $\left(k_{\mathrm{a}}\right)$ of the wellbore trajectory control tool is as follows:

$$
k_{\mathrm{a}}=\frac{180^{2} L_{\mathrm{t}}^{2} L_{3}(\omega-\Delta y)}{\pi L_{\mathrm{b}}\left(3 L_{\mathrm{b}}^{2}+2 L_{\mathrm{a}} L_{\mathrm{b}}-3 L_{\mathrm{t}}^{2}\right)\left(L_{1}+L_{2}+L_{3}\right)\left(L_{2}+L_{3}\right)}
$$

\subsection{Radial displacement caused by the combined bearing load}

According to the established static model of the tool spindle, the combined bearing mainly bears a radial force $\left(F_{\mathrm{b}}\right)$. Because the spherical thrust roller bearing is subjected to a centrifugal force during operation, the inner and outer rings can easily separate. Therefore, a given axial preload needs to be applied during work. The combined bearing is axially preloaded by compressing the left and right ends in Fig.2 of the disk springs during assembly. When the spindle deflects, in order to ensure close contact between the combined bearing and spindle, the height of the bearing washer in the combined bearing is less than the height shown in Fig. 2. Thus, the centers of the outer ring raceway do not coincide for the two ends of the spherical thrust roller bearing. $\mathrm{O}_{1}$ and $\mathrm{O}_{2}$ represents the center of outer ring raceway of left and right spherical thrust roller bearing. $\mathrm{O}$ represents the center of outer ring raceway of self-aligning roller bearing. Under the condition of spindle is deflected by $\gamma$ angle, if the outer ring of the left spherical thrust roller bearing is fixed, the intersection point between the tool spindle axis and the left outer ring spherical surface is E'. In the same way the intersection point between the tool spindle axis the right outer ring spherical surface is $\mathrm{F}^{\prime}$. With this structure, when the spindle deflects, the geometric relationship indicates that the radial displacement $(\Delta s)$ of the bearing washer causes it to "bite" into the spindle to ensure the effective transmission of force, as shown in Fig.5.
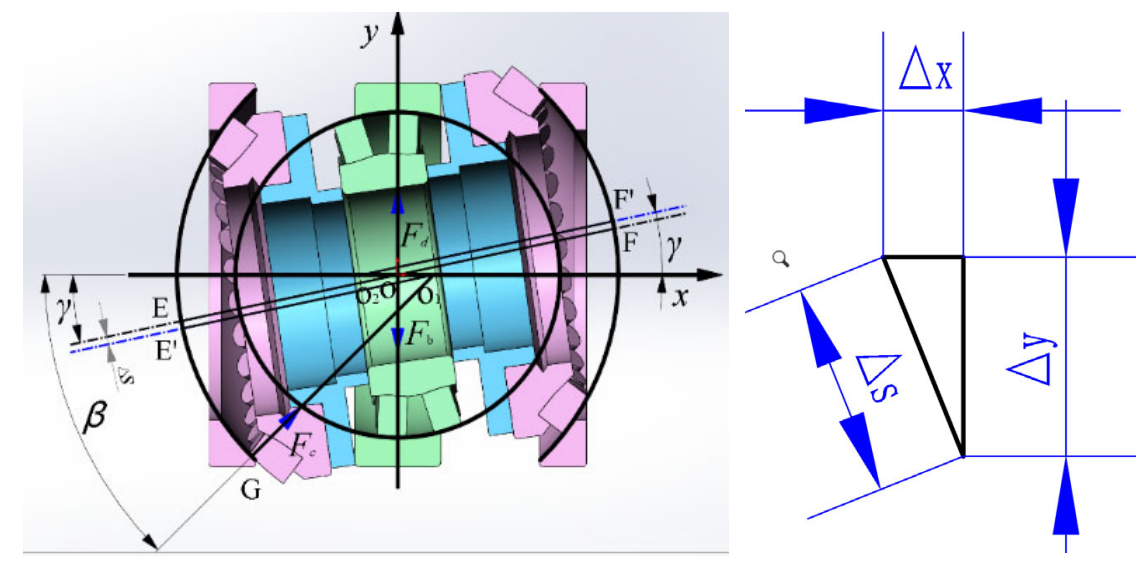

Fig. 5 Working principle of the combined bearing. 
Because the deflection angle $(\gamma)$ is very small, its influence on the load distribution can be ignored. The load at both ends of the thrust spherical roller bearings can be assumed to be symmetric. For a rolling element, the influence of the friction and moment can be ignored. Consequently, the other parts inside the combined bearing are regarded as rigid bodies. The radial force $\left(F_{\mathrm{b}}\right)$ causes the radial displacement $(\Delta y)$ of the combined bearing. This pushes the outer ring of the thrust self-aligning roller bearing at both ends of the combined bearing outward for $\Delta x$, which generates the reverse load $\left(F_{\mathrm{c}}\right)$ at the same time. The self-aligning roller bearing is also affected by the radial force, which results in the reverse support force $\left(F_{\mathrm{d}}\right)$. For the y-axis direction of the combined bearing, the force balance equation is as follows:

$$
2 F_{\mathrm{c}} \sin \beta+F_{\mathrm{d}}-F_{\mathrm{b}}=0
$$

where $\beta$ is the contact angle between the roller of the thrust self-aligning roller bearing and axial direction of combined bearing under load free condition $\left({ }^{\circ}\right)$.

According to mechanical design manual (Wen Bangchun,2010) the load deformation relation of the disk spring $\left(F_{\mathrm{c}}\right)$ is given by:

$$
F_{\mathrm{c}}=\frac{4 E_{1} t^{3} \delta}{\left(1-\mu^{2}\right) K_{1} D^{2}}\left[\frac{\left(h_{0}-\delta\right)\left(2 h_{0}-\delta\right)}{2 t^{2}}+1\right]
$$

where $\mathrm{E}_{1}$ is the elastic modulus of the disk spring (Pa), $t$ is the disk spring thickness (mm), $\mu$ is Poisson's ratio for the disk spring, $D$ is the outside diameter of the disk spring $(\mathrm{mm}), h_{0}$ is the flattening deformation of the $\operatorname{disk}$ spring $(\mathrm{mm}), \quad K_{1}$ is the calculation coefficient given by $K_{1}=\frac{1}{\pi} \cdot \frac{[(C-1) / C]^{2}}{(C+1) /(C-1)-2 / \ln C}$ $C=D / d, d$ is the inner diameter of the disk spring (mm), and $\delta$ is the deformation of the disk spring (mm).

Because the two ends of the combined bearing need to be preloaded, this produces a certain pre-compression quantity (S). Consequently, $\delta=S+\Delta x$. The raceway of the outer ring of the thrust self-aligning roller bearing is spherical. The geometric relationship can be used to derive

$$
\Delta x=R\left(\cos \beta-\sqrt{1-\left(\sin \beta+\frac{\Delta y}{R}\right)^{2}}\right)
$$

where $R$ is the outer ring raceway diameter of the spherical thrust roller bearing $(\mathrm{mm})$.

The self-aligning roller bearing in the combined bearing can be regarded as bearing a purely radial load. According to the maximum rolling load estimation formula proposed by Stribeck(Stribeck,1907)and bearing displacement estimation formula proposed by Palmgren(Palmgren,1959), the relation between the radial force $\left(F_{d}\right)$ and radial displacement for the self-aligning roller bearing is given by 


$$
\mathrm{F}_{\mathrm{d}}=\frac{Z \cos \alpha}{4.08} 0.9 \sqrt{\frac{\left(\Delta y-\Delta y_{1}\right) k^{0.8} \cos \alpha}{7.68 \times 10^{-5}}}
$$

where $Z$ is the number of rollers in the self-aligning roller bearing, $a$ is the contact angle between the roller bearing and outer ring in the self-aligning roller bearing $\left(^{\circ}\right)$, as shown in Fig.6, $\Delta y_{1}$ is the radial clearance of the selfaligning roller bearing $(\mathrm{mm})$, and $k$ is the effective length(Effective contact length except chamfering)of the roller body of the self-aligning roller bearing $(\mathrm{mm})$.

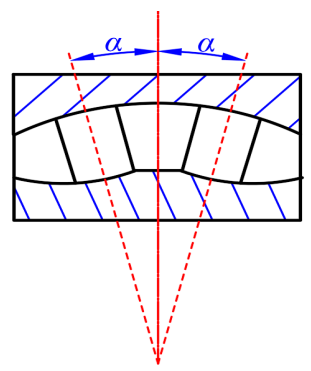

Fig. 6 Diagram of self-aligning roller bearing contact angle.

The radial displacement ( $\Delta y$ ) of the combined bearing can be obtained from simultaneous Eq. (9), Eq. (9) and Eq. $(10)-(12)$.

\section{Calculation results and analysis}

\subsection{Influence of the combined bearing displacement on the geometric deflection rate}

Since the buildup rate calculated by the model is not a real well trajectory buildup rate, in order to distinguish it from the real buildup rate, we call the build slope calculated by the model as geometric deflection rate. The relationship between the geometric deflection rate and combined bearing load is shown in Fig.7. When the wellbore trajectory control tool is inclined, the combined bearing load at the lower part increases with the inclination. This is because the deflection rate of the wellbore trajectory control tool is controlled by the maximum deflection of the spindle. Bending of the spindle increases the load on the combined bearing. The relationship between the radial displacement of the combined bearing and the buildup rate is shown in Fig.8. As the bearing's radial displacement increases, the actual rotation angle of the combined bearing decreases, which reduces the geometric deflection rate.

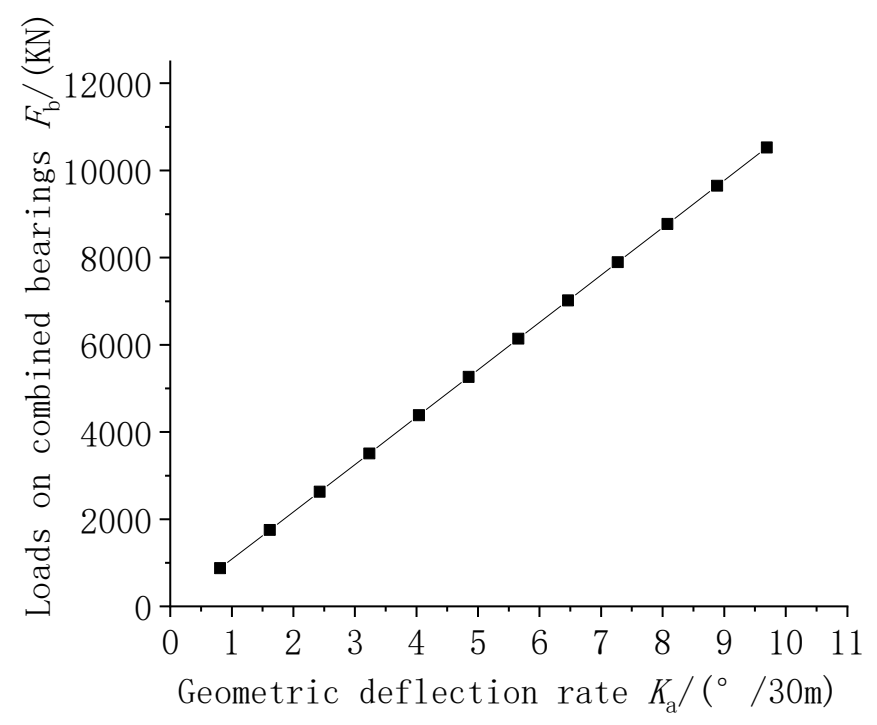

Fig.7 Relationship between the geometric deflection rate and combined bearing load. 


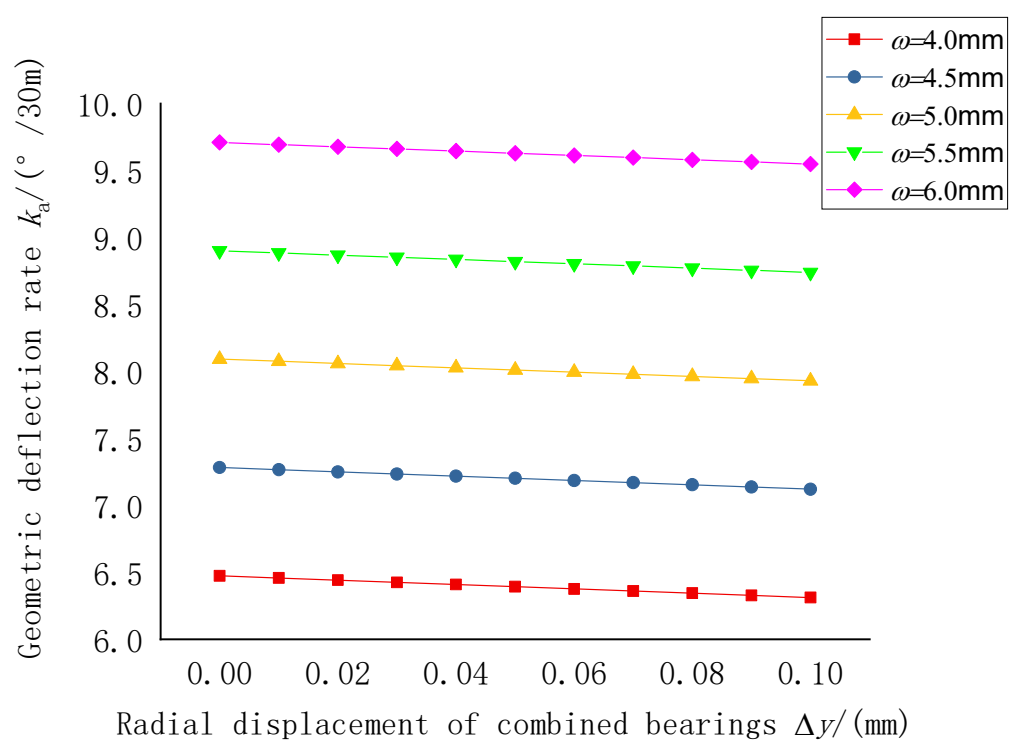

Fig. 8 Relationship between the radial displacement of the combined bearing and the geometric deflection rate.

\subsection{Influence of structural parameters for the combined bearing on the geometric deflection rate 3.2.1 Thickness of the disk spring}

The relationship between the thickness of the disk spring and the geometric deflection rate is shown in Fig.9. When the same load is applied, a smaller thickness for the disk spring increases the deformation, which weakens the support capacity for the spindle. When the thickness is insufficient, the radial displacement is large, and the buildup rate may even be negative. As the thickness of the disk spring increases, the correction of the geometric deflection rate becomes better.

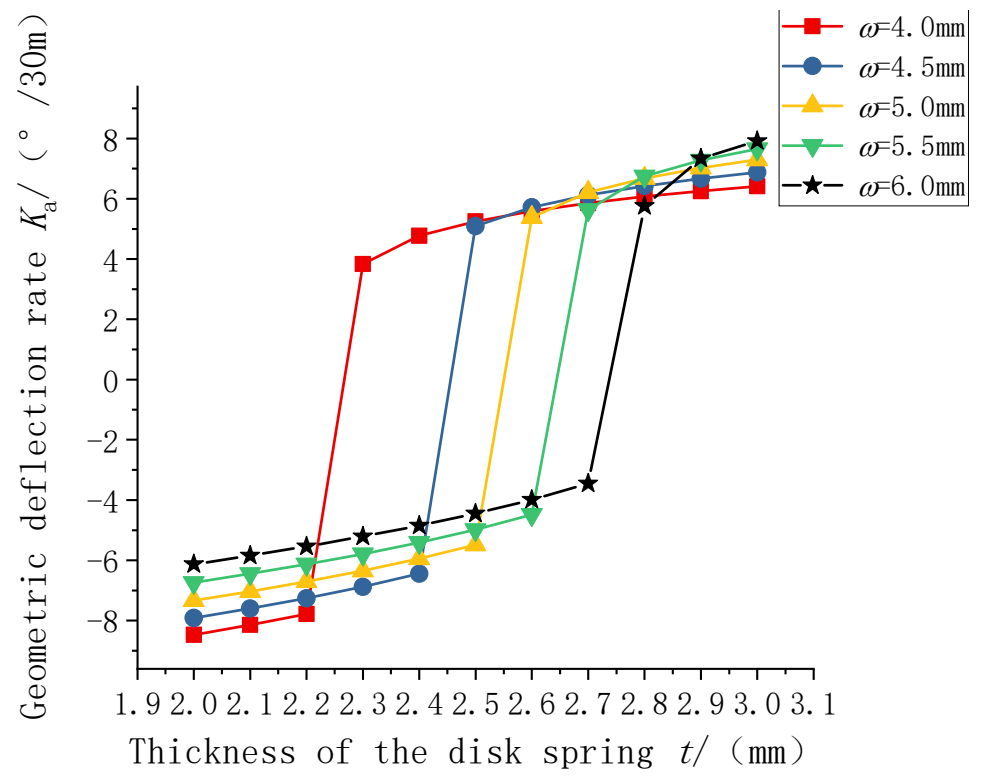

Fig.9 Relationship between the disk spring thickness and the geometric deflection rate.

\subsubsection{Pre-compression of the disk spring}

The relationship between the pre-compression quantity of the disk spring and the geometric deflection rate is shown in Fig.10. When the pre-compression of the disk spring is low, the spherical thrust roller bearings at both ends of the combined bearing are pushed far away. This increases the radial displacement of the combined bearing and reduces the geometric deflection rate. When the pre-compression amount is sufficiently large, the load cannot push the combined 
bearing away. The combined bearing is then in an ideal working state; the radial displacement caused by the load is zero, and the buildup rate reaches its maximum. However, this causes the spherical thrust roller bearing to bear a large load and affects the bearing life. Therefore, the bearing life needs to be considered when determining the amount of compression.

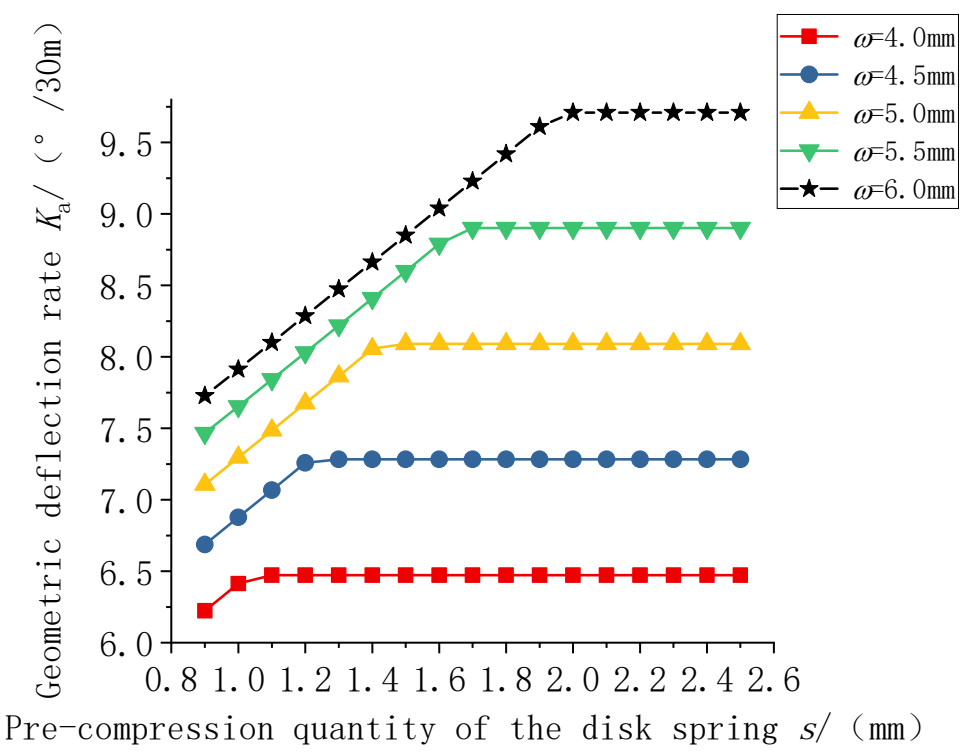

Fig.10 Relationship between the pre-compression quantity of the disk spring and the geometric deflection rate.

\subsubsection{Ratio between the internal and external diameters of the disk spring}

The relationship between the internal and external diameter ratio of the disk spring and the geometric deflection rate is shown in Fig.11. The geometric deflection rate decreases as the internal and external diameter ratio increases, which is mainly because the ratio of the disk spring is related to its deformation. Under the same load condition, a larger ratio increases the deformation, which increases the radial displacement of the combined bearing and reduces the buildup rate.

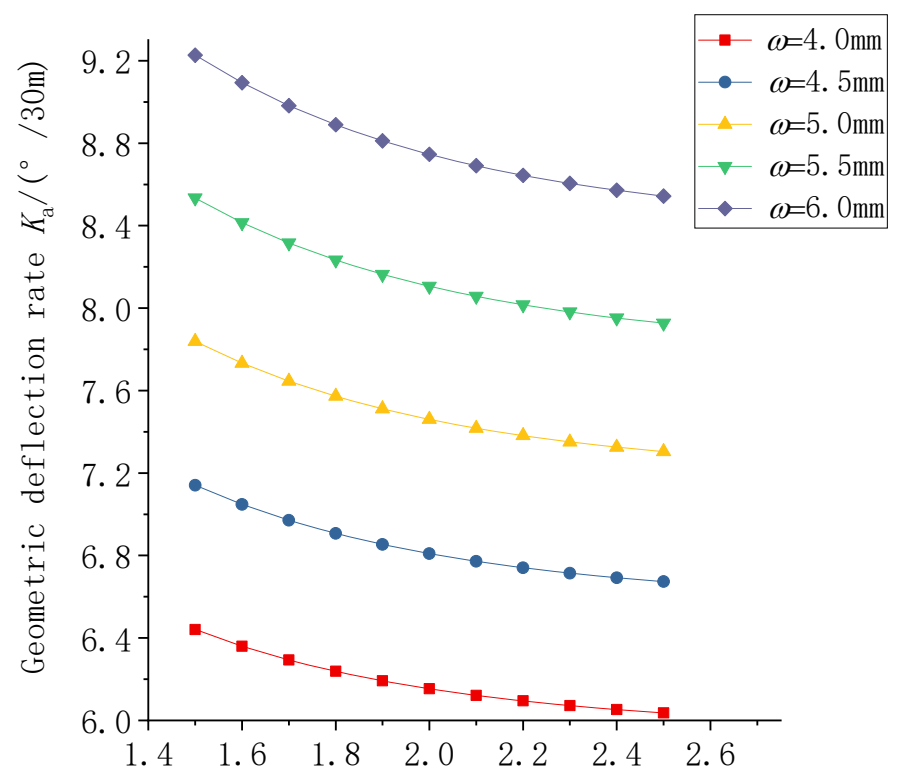

Internal and external diameter ratio of Disk spring $C$

Fig.11 Relationship between the internal and external diameter ratio of the disk spring and the geometric deflection rate. 


\subsubsection{Radial clearance of the self-aligning roller bearing}

The relationship between the radial clearance of the self-aligning roller bearing and the geometric deflection rate is shown in Fig.12. The geometric deflection rate increases with the radial clearance because the latter directly affects the radial displacement of the combined bearing. A greater radial clearance increases the radial displacement of the combined bearing and reduces the buildup rate. However, a smaller radial clearance makes it more difficult to assemble the combined bearing. In general, the radial clearance should be above $30 \mu \mathrm{m}$.

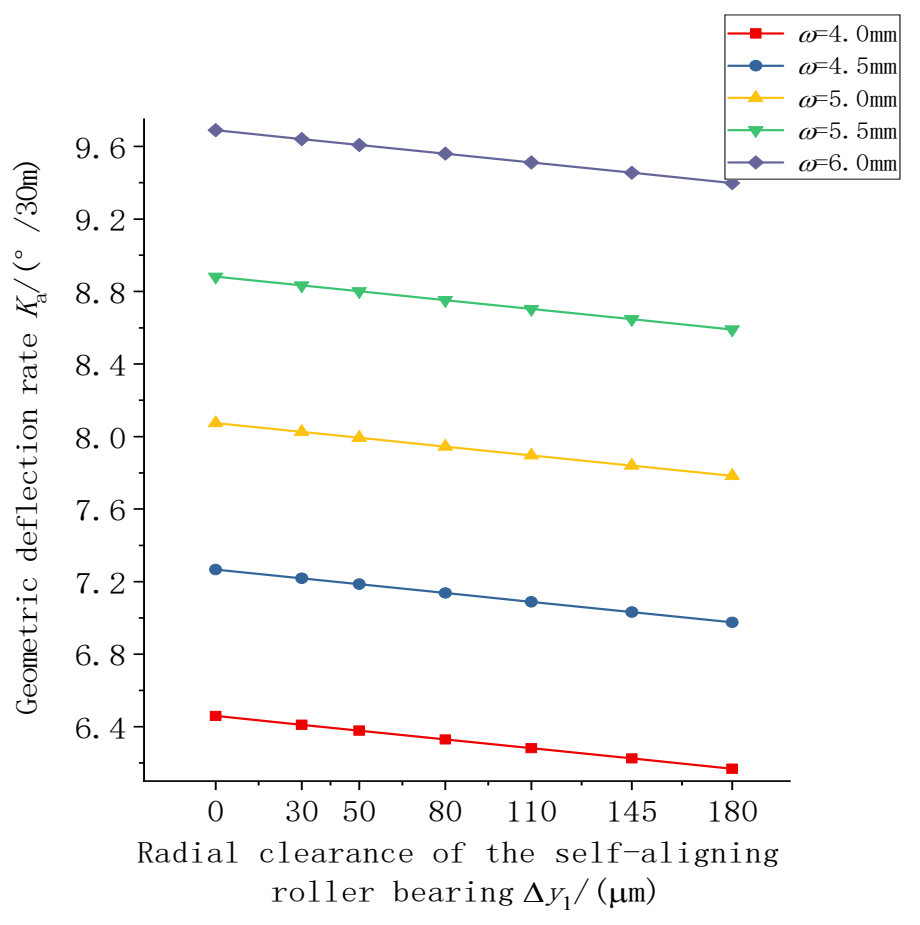

Fig.12 Relationship between the radial clearance of the self-aligning roller bearing and the geometric deflection rate.

\subsection{Factors influencing the geometric deflection rate}

The developed theoretical calculation formula for the geometric deflection rate of a wellbore trajectory control tool mainly considers the influence of the BHA, internal structural dimensions of the tool, eccentricity capacity, and radial displacement of the combined bearing. To calculate the radial displacement of the combined bearing, the structural parameters of the disk spring (thickness, pre-compression, and ratio between the inner and outer diameters), spherical thrust roller bearing (contact angle and, outer raceway circle diameter) and self-aligning roller bearing (number of rolling elements, contact angle, radial clearance, and effective length of rolling elements) are mainly considered. Each factor has a different impact on the geometric deflection rate of the wellbore trajectory control tool. In general, the main factors that affect the radial displacement of the combined bearing are the structural parameters of the disk spring and radial clearance of the self-aligning roller bearing. These four factors have different degrees of influence on the geometric deflection rate. A greater radial clearance and ratio between the internal and external diameters of the disk spring reduce the buildup rate. A greater disk spring thickness and pre-compression quantity increase the buildup rate. Because of the small space inside the tool and requirements for service life, the above parameters need to be selected while considering the space and bearing load requirements.

\section{Experiment on the buildup rate of the wellbore trajectory control tool}

Fig.13 shows a self-developed device for testing the deflection rate of the wellbore trajectory control tool. The wellbore trajectory control tool was installed on a bench with a V-shaped base and fixed and leveled by a clamping and locating device. A brushless DC geared motor was used to drive the tool spindle through a coupling. A laser emitting device was installed at the end of the tool spindle, and the axis of the spindle was directed to the laser target plate. The buildup rate was obtained by determining the position of the light spot on the target disk. The length of the tool (e.g., a 
drill bit) was simulated by adjusting the distance between the laser target disk and laser emitting device. The dimensions of the combined bearing and tool structure are given in Table 1.
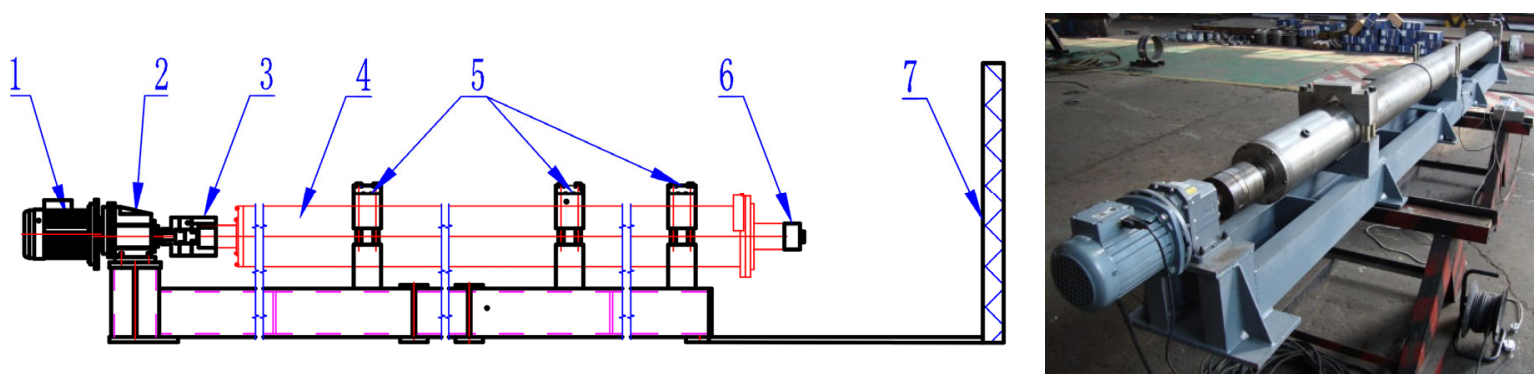

Fig.13 experiment bench for the buildup rate of the wellbore trajectory control tool: (1) motor and reduction gear, (2) tachometer and control device, (3) coupling, (4) wellbore trajectory control tool, (5) clamp and location device, (6) laser emitting device, and (7) laser target plate.

Table 1 Structural parameters and dimensions of the combined bearing and tool.

\begin{tabular}{l|c}
\hline \hline Parameter & Value \\
\hline Thickness of the disk spring $t(\mathrm{~mm})$ & 3.8 \\
Pre-compression quantity of the disk spring $S(\mathrm{~mm})$ & 1 \\
Outer ring raceway diameter of the spherical thrust roller bearing $R(\mathrm{~mm})$ & 40.21 \\
Contact angle of the spherical thrust roller bearing $\beta\left({ }^{\circ}\right)$ & 140 \\
Outside diameter of the disk spring $D(\mathrm{~mm})$ & 72 \\
Inner diameter of the disk spring $d(\mathrm{~mm})$ & 4.9 \\
Deformation of the disk spring during load free condition $h_{0}(\mathrm{~mm})$ & 18 \\
Number of rollers in the self-aligning roller bearing $Z$ & 10.2 \\
Contact angle of the self-aligning roller bearing $a\left({ }^{\circ}\right)$ & 11.3 \\
Effective length of the roller element for the self-aligning roller bearing $k(\mathrm{~mm})$ & 0.03 \\
Radial clearance of the self-aligning roller bearing $\Delta y_{1}(\mathrm{~mm})$ & 64 \\
Tool spindle diameter $D_{a}(\mathrm{~mm})$ & 2.186 \\
Distance from the upper bearing to the combined bearing $L_{t}(\mathrm{~m})$ & 1.596 \\
Distance from the elbow point to the upper bearing $L_{\mathrm{a}}(\mathrm{m})$ & 0.59 \\
Distance from the elbow point to the combined bearing $L_{\mathrm{b}}(\mathrm{m})$ & 0.8 \\
Distance from the center of the bit to the center of the lower stabilizer $L_{1}(\mathrm{~m})$ & 0.688 \\
Distance from the bent elbow to the center of the lower stabilizer $L_{2}(\mathrm{~m})$ & 1.770 \\
Distance from the center of the upper stabilizer to the bent elbow $L_{3}(\mathrm{~m})$ & \\
\hline
\end{tabular}

Table 2 compares the test and calculation results as the spindle deflection of the control tool was varied under the fixed conditions of a spindle speed of $60 \mathrm{rpm}$ and ambient temperature of $21^{\circ} \mathrm{C}$. Theoretical results without considering the influence of combined bearings was giving by direct use of Eq. (2). The results showed that the calculated buildup rate was very close to the experimental results. In particular, when the maximum deflection of the main shaft exceeded $4 \mathrm{~mm}$, the calculation error of the buildup rate considering the radial displacement of the combined bearing was less than $1 \%$. The results suggest that the radial clearance of the combined bearing has a significant effect on buildup rate, especially when the spindle deformation is less than $4 \mathrm{~mm}$. If the spindle deformation continues to increase, might lead to the closes of gap clearance, which will result in full contact between the combined bearing and the spindle. Under such circumstances, the disc spring is deformed under axial load, and the radial displacement of the combined bearing increases further, which will directly cause the buildup rate less than the theoretical results that without considering the combined bearings. However, the above inference is only based on the research results of this paper and has not been 
verified by experiments. In order to further clarify the influence of radial clearance on buildup rate, it is necessary to further study the inner ring motion of combined bearing. Meanwhile, in the following research, it is still necessary to design and carry out the combined bearing inner ring movement detection experiment.

Thus, in the case of a large deflection of the main shaft, the proposed calculation method for the buildup rate is highly accurate and can meet the accuracy requirements of projects. In addition, the proposed method for calculating the geometric deflection rate intuitively reflects the influence and function of the structural parameters of the combined bearing. Thus, it is of great significance to the design of combined bearings for tools.

Table 2 Experimental results.

\begin{tabular}{|c|c|c|c|c|c|}
\hline \multirow{2}{*}{$\begin{array}{l}\text { Maximum deflection } \\
\text { of the spindle } \\
\omega(\mathrm{mm})\end{array}$} & \multicolumn{3}{|c|}{ Buildup rate $(\% / 30 \mathrm{~m})$} & \multicolumn{2}{|c|}{ Error $(\%)$} \\
\hline & $\begin{array}{l}\text { Theoretical results } \\
\text { without considering the } \\
\text { influence of combined } \\
\text { bearings }\end{array}$ & \begin{tabular}{|lr} 
Theoretical results \\
considering the \\
influence of combined \\
bearings
\end{tabular} & $\begin{array}{l}\text { Test } \\
\text { results }\end{array}$ & $\begin{array}{l}\text { Without the } \\
\text { combined } \\
\text { bearings }\end{array}$ & $\begin{array}{l}\text { With the } \\
\text { combined } \\
\text { bearings }\end{array}$ \\
\hline 1 & 1.618 & 1.566 & 1.589 & 1.87 & -1.44 \\
\hline 2 & 3.237 & 3.181 & 3.289 & -1.60 & -3.31 \\
\hline 3 & 4.855 & 4.796 & 4.693 & 3.46 & 2.20 \\
\hline 4 & 6.473 & 6.411 & 6.349 & 1.96 & 0.98 \\
\hline 5 & 8.092 & 8.026 & 7.981 & 1.39 & 0.57 \\
\hline 6 & 9.710 & 9.641 & 9.631 & 0.82 & 0.11 \\
\hline
\end{tabular}

\section{Conclusion}

(1) The combined bearing of the tool is mainly subjected to a radial load, which causes a radial displacement that is related to the structural parameters of the bearing. The buildup rate increases with a greater thickness and pre-compression quantity of the disk spring inside the combined bearing and with a decreasing radial clearance of the self-aligning roller bearing and ratio between the inner and outer diameters of the disk spring.

(2) When the spindle deflection is small, the combined bearing bears a small load, which has little impact on the buildup rate. When the deflection is large, the combined bearing bears a large load, which causes radial displacement and reduces the buildup rate. For the wellbore trajectory control tool in this study, the combined bearing had a significant effect on the buildup rate when the maximum deflection of the main shaft reached more than $4 \mathrm{~mm}$.

(3) Increasing the disk spring thickness and pre-compression quantity helps increase the buildup rate, but this also sharply increases the load on the spherical thrust roller bearing, which affects the life and reliability of the tool. Thus, the spring thickness and pre-compression quantity need to be set to reasonable values to ensure a given service life.

(4) Reducing the radial clearance of the self-aligning roller bearing increases the buildup rate but makes assembling the combined bearing more difficult. In general, the radial clearance should be more than $30 \mu \mathrm{m}$.

\section{Acknowledgements}

This work of the paper was supported by the scientific research program of Hubei education apartment(Q20181315)and Hubei Technology Innovation Project (major project) (2019AAA010).

\section{References}

Dong, C., Dupuis, C., Morriss, C., Legendre, E., Mirto, E., Application of automatic stochastic inversion for multilayer reservoir mapping while drilling measurements, Abu Dhabi International Petroleum Exhibition and Conference, Society of Petroleum Engineers (2015).

Feng, D., Lu, C., Zhang, H., Huang, K., Research on Control of Steering Movement of Well Trajectory Control Tool, Oil Field Equipment, Vol.46, No.1 (2017),pp. 6-10. 
Gong, F. X., Huang X. Y.,Predict and control method of well path for push the bit RSS, Science Technology and Engineering, Vol.18 (2016),pp. 20-4 .

Hong, D., Tang, X., Su Y, Shen, L., Dou, X., Generalized beam-column method for non-continuous rotary steering drilling of bottom-hole assembly, Acta Pet $\operatorname{Sin}(2014)$,pp.543-50.

Hassen, B. R., MacDonald, A. J., Field comparison of medium and long radius horizontal wells drilled in the same reservoir,In: SPE/IADC Drilling Conference, Richardson, TX: SPE(1990), pp. 637-52.

Karlsson, H., Brassfield, T., Performance drilling optimization, In: SPE/IADC Drilling Conference, Richardson, TX: SPE(1985), pp. 439-46.

Karlsson, H., Cobbley, R., Jaques, G. E.,New developments in short-, medium-, and long-radius lateral drilling,In: SPE/IADC Drilling Conference, Richardson, TX: SPE(1989), pp. 725-36.

Kong ,D. L., Li, Q., Optimization of borehole trajectory with multi-control points for long lateral section horizontal wells, China Offshore Oil and Gas, Vol.28,No.5 (2016),pp.92-97.

Liu, X.,Practical calculation method of geometric deflection rate of guide drilling tool, Nat Gas Ind 11(2005), pp.50$52+150$.

Liu, X., He, S., Zou, Y. (2004). Study on the geometric build angle rate of steerable motor. Acta Pet Sin, 6, 83-7.

Li, Y., Shen, Q. Y., Lu, H., Wang, B. H. M., Fu, X., Chen, J. F., Inversion and uncertainty assessment of ultra-deep azimuthal resistivity logging-while-drilling measurements using particle swarm optimization. Journal of Applied Geophysics(2020), p.178.

Li, C. L., Application study on rotary steering drilling technology and its drilling tool. Oil Field Equip, Vol. 43 ,No. 9 (2014) pp. 69-73.

Minett-Smith, DJ, Stroud, DRH, Lines, L. A. Pagett, JM., Novel detection system improves understanding of backwards whirl and aids parameter optimization for advanced oilfield Rotary Steerable Drilling Systems, In: 10th International Conference on Vibrations in Rotating Machinery(2012), p.601-611.

Shi, L., Wang K. Q., Feng D., Zhang H., Wang P., Wellbore trajectory control tool seal system leakage analysis based on steady gap flow, Advances in Mechanical Engineering, Vol.12, 168781402093046 (2020).

Shi, L., Shuai, J., Xu K.,. Fuzzy fault tree assessment based on improved AHP for fire and explosion accidents for steel oil storage tanks. Journal of Hazardous Materials(2014), p.278.

Su, Y., Tang X., Gal L., Problem of double and triple bent PDM equivalent to single bent PDM,Acta Pet Sin, Vol.23,No. 2(2002), pp.77-81.

Wang, B., Xu, D., Cheng, C., Geometric analysis and calculation of build-up rate of motor drilling tool, Oil Drill Prod Technol, Vol.16,No.1(1994),pp.32-7.

Wang, Y., Zhang, L., Tang, N., Huo, A., Cheng W., Dynamics and rotational movement analysis of servo platform for rotary steerable drilling system, J Mech Eng,Vol.17(2012),pp.65-69.

Zhang, G. W., Zhan, M. L., Design of a new full rotation and dynamic point-the-bit rotary steerable drilling system,China Petrol Mach, Vol.44 ,No.5 (2016), pp. 13-17.

Wen, Bangchun, Mechanical Design Manual, Machinery Industry Press,2010, pp. 11-76.

Stribeck, R., Ball bearings for various loads, ASME 29(1907), pp. 420-463.

Palmgren, A., Ball and Roller bearing Engineering, $3^{\text {rd }}$ ed., Philadephia(1959), pp. 1-14. 